\title{
COMMUNICATIONS
}

\section{RETINAL PHOTOGRAPHY IN COLOUR}

BY

\author{
PETER HANSELL AND E. J. G. BEESON \\ Medical Illustration Dept., Institute of Ophthalmology, London, and the Research Laboratory, \\ British Thomson-Houston Co., Ltd.
}

HisTORICAL.-Monochrome photography of the human retina has been practised increasingly since the early pioneer work on this subject in the latter half of the 19th century. The instrument in most common use to-day is the Zeiss-Nordenson retinal camera, which first appeared in 1915. Several modifications of the original instrument have since appeared, and over 10 years ago it was reported that about 350 of these cameras had been distributed by the manufacturers (Koch, 1941). The early models employed a carbon arc light source, and later an incandescent lamp was used. The general appearance of the apparatus is shown in Fig. 1.

Colour photography of the ocular fundus has been developed relatively recently; early results on Dufaycolor and Agfacolor were hardly satisfactory, but Kodachrome began to be used successfully about 1935 .

Many collections of Kodachrome transparencies have by now been made, and the Zeiss-Nordenson carbon-arc model has been favoured for this purpose. It is also interesting to note that, after much research, the Bausch and Lomb Co. have reverted to the use of a carbon-arc in their new retinal camera (Med. biol. Illus., 1952, 2, 58).

Various workers (Bedell, 1940; Koch, 1941) have reported that exposure times of $\frac{1}{2}-\frac{1}{12}$ sec. are necessary to obtain results with this type of subject and equipment. With exposures such as these, there is a considerable risk of records being marred by movement of the subject, and the unstable nature of an open carbon arc makes serial recording unpredictable. These diffi-

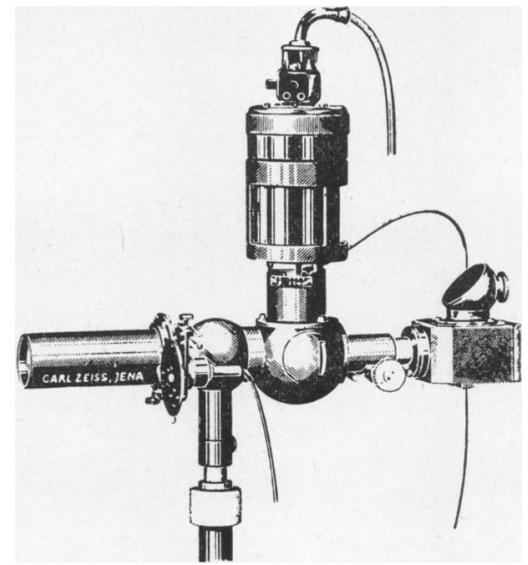

Fig. 1.-General appearance of ZeissNordenson retinal camera. 
culties chiefly encouraged us to look for an improved light source and determined the final choice.

\section{DeVelopment OF THE FA5 XeNON LAMP}

The present work has been concerned with the development of a new light source to be used with a Zeiss-Nordenson camera for colour photography of the retina. The basic requirements were taken to be:

(i) The illumination should be sufficiently constant to allow alignment and focusing of the optical system and visual examination of the retina without discomfort to the patient.

(ii) The light-source should be stable, constant, and of a spectral emission suited to colour-film sensitivity.

(iii) The illumination should be greatly increased for the actual moment of photographic exposure. To minimize discomfort and involuntary movement and blinking on the part of the patient $\frac{1}{25} \mathrm{sec}$. should be the maximum permissible exposure.

A dimensioned drawing of an experimental lamp (BTH, FA5) is given in Fig. 2. The first requirement can be met by operating this lamp as a continuous arc at a current of 10 amps with a potential of 15 volts.

The second requirement is achieved by filling the glass envelope of the

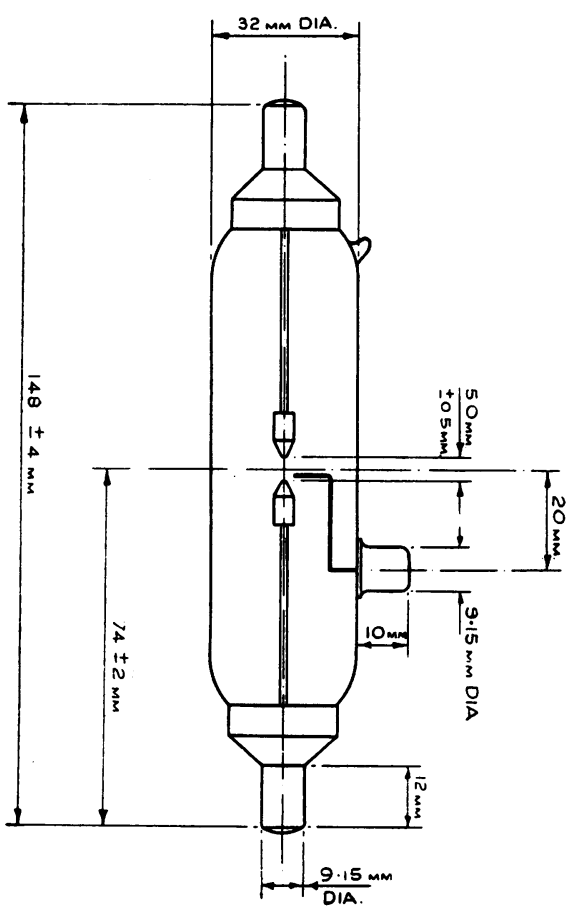

FIG. 2.-British Thomson-Houston FA5 xenon arc lamp (flash tube). lamp with pure xenon which gives a spectral emission approximating daylight.

The first line of approach to the third problem, that of providing intense illumination for exposure, was to run the lamp as a continuous arc for focusing, and then to switch off and pass a condenser charge through the tube in the same manner as an "electronic flash". Tests revealed several disadvantages, the first of which was the length of time required to allow the electrodes to cool sufficiently to prevent autotriggering of the condenser flash, and the noise produced by the sudden discharge was another undesirable feature. The main reason for abandoning this method, however, was that, with increasing energy, the light source increased in size rather than intrinsic brightness. As there 
are two limiting apertures in the system-one in the camera and the other the pupil of the eye-nothing could be gained from a larger source; adequate results were obtained on fast monochromatic film, but the effective light was insufficient to expose colour film.

Another possibility was that of over-running or pulsing the lamp at a high current for an instant during its normal running as a $150 \mathrm{w}$ continuous arc. Tests made with a compact source mercury discharge lamp (Type ME) and a slow monochromatic film showed that the xenon lamp would require to be pulsed at some 5,000 w to achieve results on colour film at an exposure of $\frac{1}{25}$ second. With the equipment to be described, it is indeed possible to overexpose Kodachrome film at this speed.

A control unit for this pulse operation has now been developed. A detailed specification is outside the scope of this paper, but full technical data are available and a simplified circuit and description are given in Fig. 3.

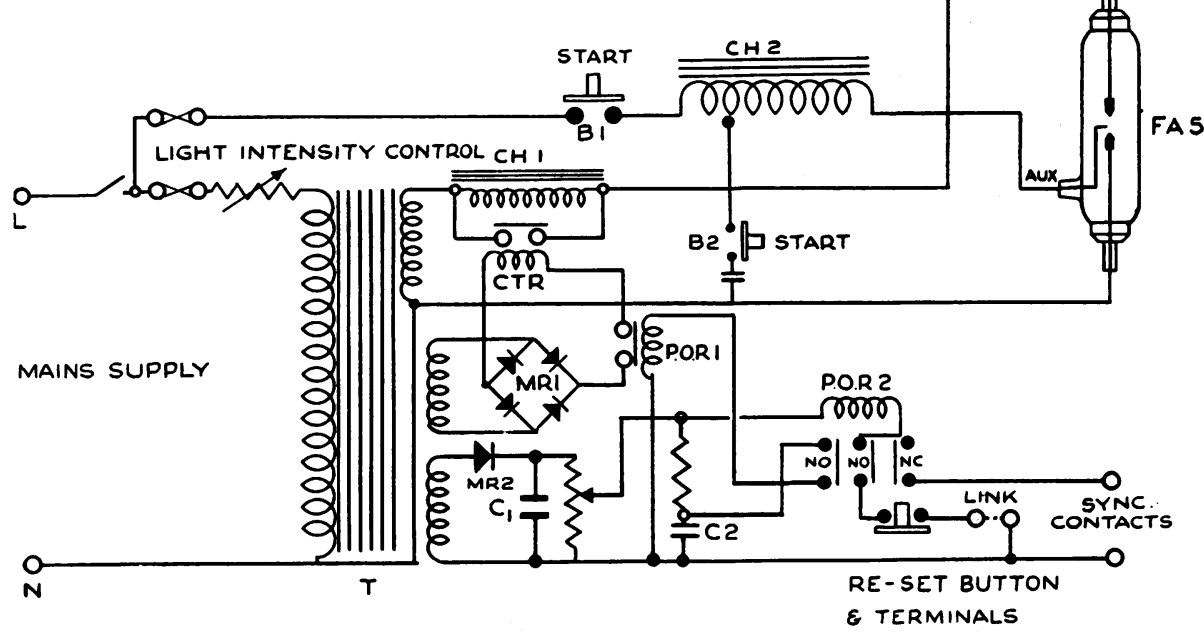

Fig. 3.-Xenon lamp pulse circuit.

The circuit design is for normal 200/250v 50-cycle AC operation providing current to the FA5 lamp at 40 volts. On closing the main switch the two main lamp electrodes are made alive. As the gas pressure in the lamp is high at room temperature, it is necessary to provide a surge of voltage from transformer $\mathrm{CH} .2$ to the auxiliary electrode on the lamp by closing push button $B_{1}$ and intermittently opening and closing the start button $\mathbf{B}_{2}$. The intermittent operation of button $\mathrm{B}_{2}$ causes a peaking 50 -cycle AC voltage to initiate the auxiliary arc. Once this arc discharge has been established by further intermittent closing and opening of $\mathbf{B}_{2} \mathbf{B}_{1}$ still being held closed, the main arc gap breaks down, and once the two main electrodes have reached a temperature high enough, the auxiliary arc is extinguished by releasing button $B_{1}$. The lamp is now running at a steady power of approx. $150 \mathrm{w}$.

To pulse the lamp, synchronizing contacts are provided which, when shorted together, close P.O. relay 2. The contacts on this relay in turn close and complete a further circuit to cause the charged condenser C2 to be discharged through P.O. relay 1. The closing of relay POR. 1 in turn closes a high speed contact CTR which momentarily allows a heavy current to flow through the lamp. Normally the current in the lamp is limited by the series choke CH.1. The value of the current in the mally the current in the lamp is limited by the series choke CH.1. The value of the current in the
lamp is controlled by the variable resistance in the primary circuit of the main transformer, and the
duration of the flash by the energy stored in the condenser C.2. and discharging this through P.O. duration of the flash by the energy stored in the condenser C.2. and discharging this through P.O.
relay 1. The timing of this circuit is pre-set to give a flash of light with a duration of 1. After each flash the circuit requires to be reset, and as well as the toggle switch provided for this purpose a link is also supplied so that automatic resetting and tripping of the circuit can be arranged independently of any manual operation.

The voltage and current necessary to trip the circuit are of such a value that a micro-relay or normal camera contacts are adequate to trip the flashing of the lamp. 
Use of THE FA5 Xenon Lamp with the ZeIss-Nordenson Camera

The lamp is of such a size that it may be mounted vertically on the ZeissNordenson camera in the position normally occupied by the carbon-arc housing (Figs 4 and 5). The electrodes can be positioned in relation to the

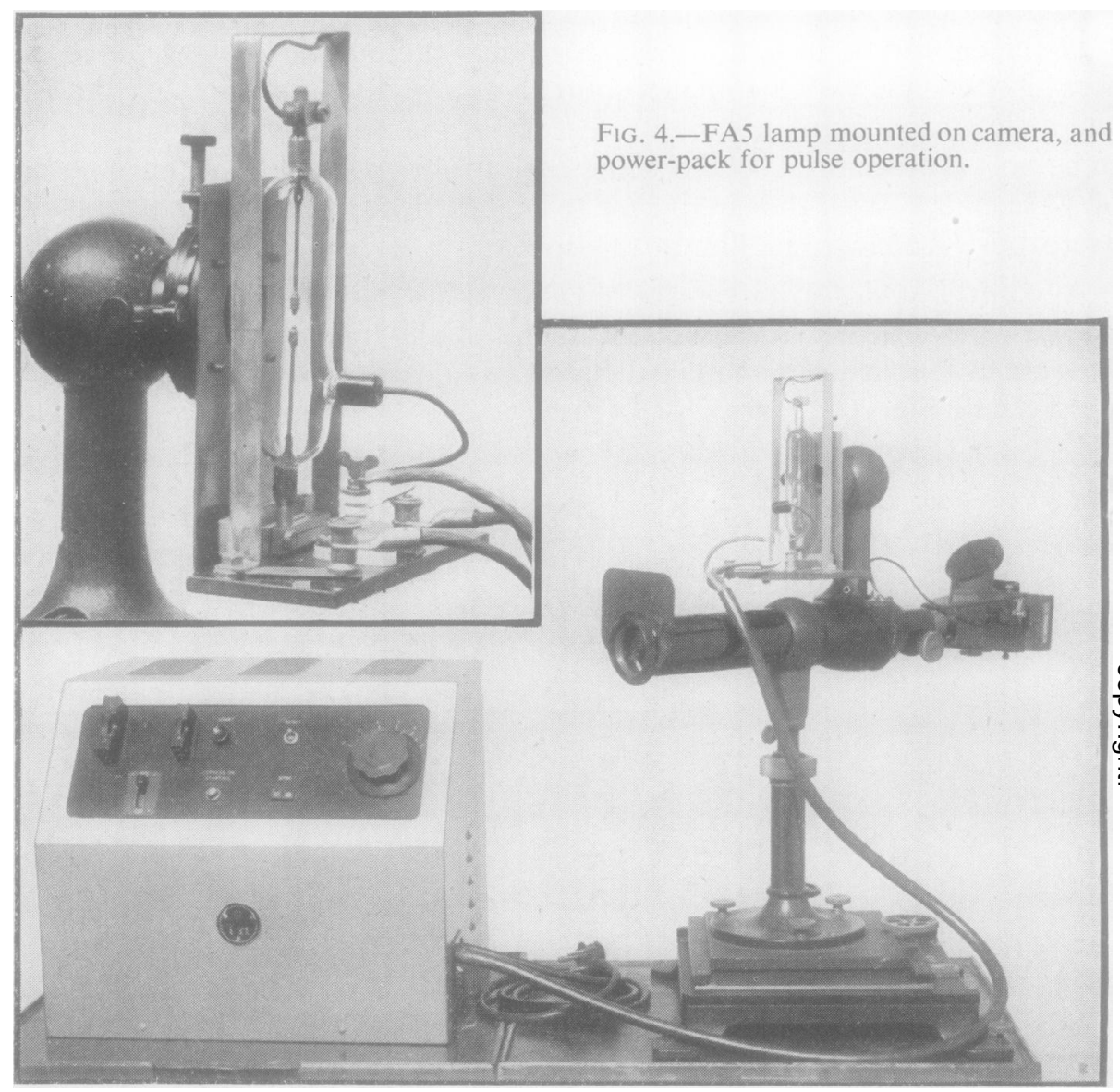

first condenser, and thereafter the vertical and horizontal slides provided on the instrument can be used for centring the arc.

The level of illumination emitted when the lamp is burning normally is lower than that of the original carbon arc, and therefore the neutral density filter in

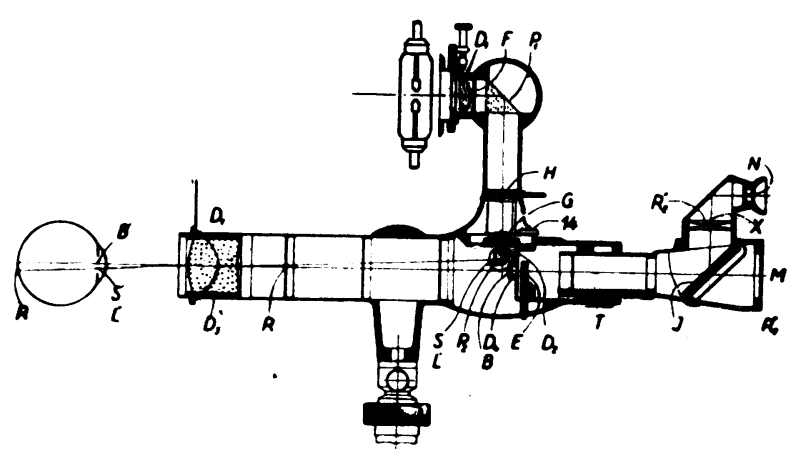

FIG. 5.-Diagram of optical system of Zeiss-Nordenson camera, showing position of FA5 lamp. 


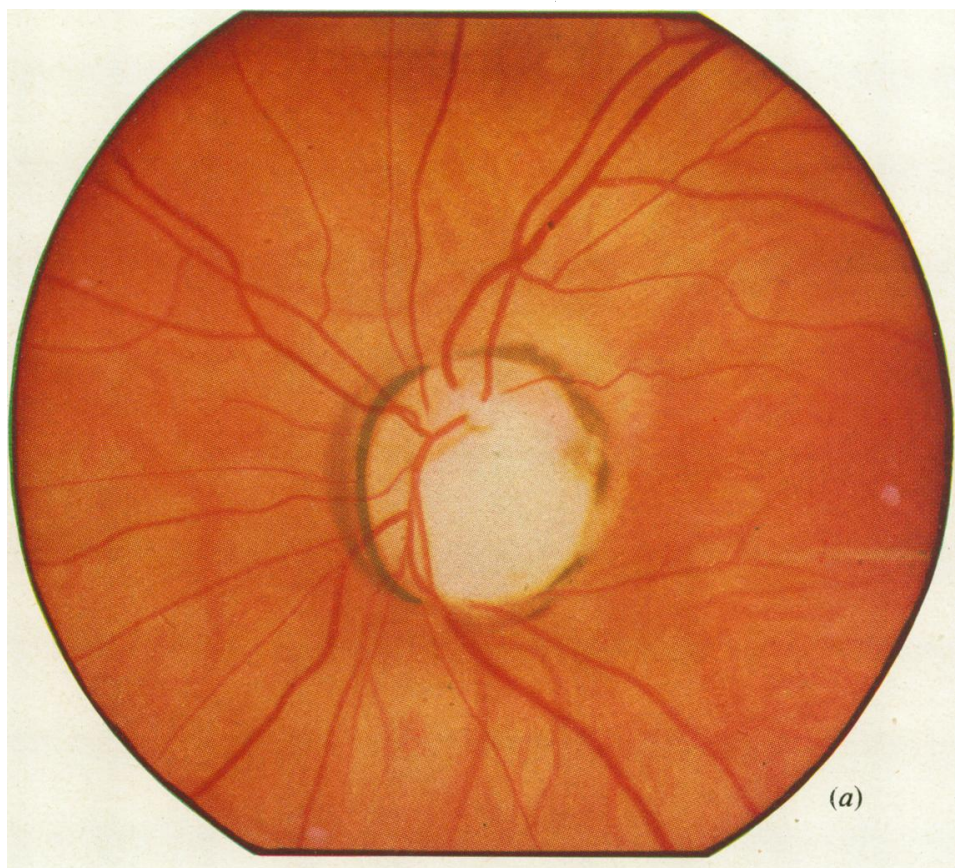

FIG. 6.-Kodachrome Bantam frames obtained at $\frac{1}{25}$ sec. with FA5 lamp.

(a) Congenital anomaly of left optic disc.

(b) Toxoplasmosis of right eye. 
the shutter must be replaced by a lighter one to provide enough transmission for visual focusing and examination of the retina.

The pulsed high-intensity illumination is limited to $\frac{1}{25} \mathrm{sec}$. by the control unit, and it is therefore necessary to synchronize this with the shutter opening. In practice it has been found convenient to trigger the pulse by mechanical contacts activated by the shutter mechanism itself. To obviate the necessity for fine control and delay mechanisms, it is advisable to fit the ${ }_{25} \mathrm{sec}$. pulse into a shutter opening of $\frac{1}{10} \mathrm{sec}$.

Finally, the camera back has been modified to accept Bantam roll film, for Kodachrome film is available in this size in eight-exposure lengths; furthermore, the picture size is slightly larger than that obtained on $35 \mathrm{~mm}$. film. A similar modification has been described by Markham (1951). Fig. 6 is a reproduction of two Kodachrome Bantam frames made with the above equipment.

OtHER APPLICATIONS.-The FA5 lamp has characteristics which make it suitable for other uses. Where constant adjustment and focusing of an optical system are required, the steady source of light together with the ability to produce a flash at a desired moment, would meet many needs in lit-lamp photography. In photomicrography and time-lapse photography of living matter the heating problem. normally encountered is considerably reduced by the very brief duration of the pulsed flash. The fact that this lamp can also be operated by condenser discharge adds to the range of its possible uses. It has already been used as a compact stroboscopic source with a repetition frequency exceeding 160 flashes per second.

\section{SUMMARY}

The application of a new compact xenon arc lamp (FA5) to retinal photography in colour is described. The circuit required for pulse operation of the lamp is given and some modifications of the Zeiss-Nordenson retinal camera are mentioned.

By these means standard and comparable records of the fundus oculi may be obtained on Kodachrome colour film at exposures of $\frac{1}{25} \mathrm{sec}$.

We should like to thank members of the staff of the Institute of Ophthalmology and of the research laboratory of the British Thomson-Houston Company for helpful advice and facilities for experiment.

\section{REFERENCES}

Bedell, A. J. (1940). In " Modern Trends in Ophthalmology", ed. F. Ridley and A. Sorsby, chap. 19, pp. 213-223. Butterworth, London.

КоCH, F. L. P. (1941). J. biol. photogr. Ass., 9, 119-135.

MARKHAM, M. (1951). Ibid., 19, 124. 